\title{
Evaluación de la exposición al polvo de carbón y de sílice en sitios de minería subterránea en tres departamentos de Colombia
}

\author{
Marcela Varona ${ }^{1}$, Milcíades Ibáñez-Pinilla ${ }^{1}$, Leonardo Briceñoํ․ Helena Groot² Diana Narváez², \\ Marién Palma ${ }^{3}$, Diego Herrera', Gloria Morgan ${ }^{4}$, Carlos Torres ${ }^{1}$ \\ Escuela de Medicina y Ciencias de la Salud, Universidad del Rosario, Bogotá, D.C., Colombia \\ 2 Laboratorio de Genética Humana, Universidad de los Andes, Bogotá, D.C., Colombia \\ 3 Grupo de Salud Ambiental y Laboral, Instituto Nacional de Salud, Bogotá, D.C., Colombia \\ 4 Positiva Compañía de Seguros S.A., Bogotá, D.C., Colombia
}

Introducción. La neumoconiosis de mineros del carbón es una enfermedad crónica e irreversible que se considera un problema de salud pública.

Objetivo. Estimar la prevalencia de neumoconiosis y sus factores asociados en mineros de carbón de Boyacá, Cundinamarca y Norte de Santander.

Materiales y métodos. Se hizo un estudio de corte transversal en 476 mineros. Se les hizo valoración médica, se tomaron radiografías de tórax siguiendo los criterios de la Organización Internacional del Trabajo, así como espirometrías, y se identificaron los polimorfismos de la glutatión S-transferasa y de las enzimas de reparación. Las asociaciones entre las condiciones ocupacionales y de la empresa con la neumoconiosis, se estudiaron usando modelos de regresión de Cox.

Resultados. En 31 empresas se hicieron 479 monitorizaciones ambientales y se evaluaron 476 trabajadores cuyo tiempo de trabajo fluctuaba entre 10 y 57 años. La prevalencia de la neumoconiosis fue de 33,8 \% ( IC $_{95 \%}$ 27,0-41,3). En el modelo de regresión multivariado de Cox, con tiempo de riesgo constante para la neumoconiosis, esta se asoció significativamente con el trabajo en empresas medianas (razón de prevalencias, $\mathrm{RP}=2,00 ; \mathrm{IC}_{95 \%}$ 0,995- 2,690; $\mathrm{p}=0,052$ ), con un nivel alto de exposición al polvo de carbón según el índice de exposición ( $R P=2,055 ; I_{95 \%} 1,043-4,048 ; p=0,038$ ), y una antigüedad de 25 años o más (para 25,0 a 29,9 años: $R P=2,199 ; I_{95 \%} 1,449-3,338 ; p=0,001$ ).

Conclusiones. La prevalencia de la neumoconiosis fue muy alta y se asoció a la exposición a altos niveles de polvo de carbón, a una exposición laboral mayor o igual a 25 años y al trabajo en empresas medianas.

Palabras clave: neumoconiosis; minas de carbón; exposición profesional; genotoxicidad; prevención de enfermedades; sílice libre.

doi: https://doi.org/10.7705/biomedica.v38i4.4183

Evaluation of the exposure to coal dust and prevalence of pneumoconiosis in underground mining in three Colombian departments

Introduction: Coal workers' pneumoconiosis is a chronic and irreversible disease representing a public health problem.

Objective: To estimate the prevalence of pneumoconiosis and its associated factors among underground coal miners in the Colombian departments of Boyacá, Cundinamarca and Norte de Santander.

Materials and methods: We conducted a cross-sectional study of 476 miners to measure the prevalence of pneumoconiosis and its associated factors such as coal dust and silica levels, as well as

\footnotetext{
Contribución de los autores:

Marcela Varona: diseño del estudio, protocolo de investigación y proyecto de investigación

Leonardo Briceño: diseño del estudio y proyecto de investigación

Helena Groot y Diana Narváez: desarrollo de las pruebas genéticas

Marién Palma: recolección de muestras biológicas y ambientales en campo

Diego Herrera: preparación general de los reportes

Milcíades Ibáñez-Pinilla: análisis estadístico, muestreo, metodología y estudio epidemiológico

Gloria Morgan: desarrollo de las pruebas para determinación en aire de polvos de carbón y de sílice

Carlos Torres: evaluaciones médicas

Todos los autores participaron en la escritura del artículo
} 
the occupational conditions. The medical assessment and a chest $\mathrm{x}$-ray were performed according to the International Labor Organization criteria, along with spirometry and the identification of glutathione S-transferase and repair enzyme polymorphisms. The associations were explored using Cox regression models.

Results: We performed a total of 479 environmental monitoring sessions in 31 companies and we evaluated 476 workers with 10 to 57 years of mining work experience. The prevalence of pneumoconiosis was $33.8 \%(95 \% \mathrm{Cl}: 27.0-41.3 \%)$. In the Cox multivariate regression model with a constant risk time, pneumoconiosis was significantly associated with working in medium-sized companies (PR=2.00, 95\% $\mathrm{Cl}: 0.995-2.690 ; p=0.052)$, the level of severe exposure to coal dust $(\mathrm{PR}=2.055,95 \% \mathrm{Cl}: 1.043$ 4.048; $p=0.038$ ), and working in underground mining for 25 years or more (for those with 25.0-29.9 years: $P R=2.199,95 \% \mathrm{Cl}: 1.449-3.338 ; p=0.001$ ).

Conclusions: The prevalence of pneumoconiosis was very high and was found to be associated with severe exposure to coal dust, work exposure for 25 years or more and working in medium-sized enterprises.

Key words: Pneumoconiosis; coal mining; occupational exposure; genotoxicity; disease prevention; free silica.

doi: https://doi.org/10.7705/biomedica.v38i4.4183

El término 'neumoconiosis' se utiliza para describir un conjunto de enfermedades pulmonares asociadas con la inhalación de un agente (polvo, humo, fibra), normalmente en un entorno laboral donde la absorción de algún componente específico es el factor causal clave (1). En el contexto específico de la neumoconiosis de los mineros del carbón, se presume una alta prevalencia debido a que la exposición ocupacional en minas subterráneas es de características desfavorables como un porcentaje estimado de polvo de carbón respirable de 40 a $95 \%$ (2); además, la media geométrica de sílice libre es superior a la de otros ambientes laborales (3).

La neumoconiosis se desarrolla después de varios años de exposición laboral y suele tardar por lo menos diez en manifestarse. En su forma más simple, generalmente no se asocia con síntomas respiratorios o signos físicos anormales, pero en su forma complicada se manifiesta con tos, disnea y, en ciertos casos, progresa a insuficiencia cardíaca derecha $(1,4)$.

No existen tratamientos médicos eficaces para la neumoconiosis, razón por la cual es importante detectarla tempranamente y reducir la exposición mediante actividades de prevención enmarcadas en un programa de vigilancia en salud que incluya pruebas periódicas de función pulmonar y radiología (1).

\section{Correspondencia:}

Marcela Varona, Escuela de Medicina y Ciencias de la Salud, Universidad del Rosario, Carrera 24 № 63-C-69, Bogotá, D.C., Colombia.

Teléfono: (571) 297 0200, extensión 3343

marcela.varona@urosario.edu.co
Las pruebas de la función pulmonar permiten evaluar la magnitud de la obstrucción bronquial difusa, uno de los componentes más significativos de la neumoconiosis, cuyo indicador más adecuado es el volumen espiratorio forzado en el primer segundo $\left(\mathrm{VEF}_{1}\right)$, empleado para demostrar la existencia de obstrucción bronquial al relacionarlo con la capacidad vital forzada (índice $\mathrm{VEF}_{1} / \mathrm{CVF}$ ) (5).

En cuanto a la radiología, se emplea la clasificación de la Organización Internacional del Trabajo (OIT), la cual se basa en la presencia de anormalidades parenquimatosas y pleurales (6), y considera la silicosis nodular, la tuberculosis miliar y la enfermedad metastásica pulmonar en el diagnóstico diferencial (7).

Por otra parte, para establecer la asociación entre los factores genéticos y la propensión a desarrollar neumoconiosis, se utiliza el estudio de posibles polimorfismos en el ADN de los trabajadores. Se ha evidenciado que la exposición al polvo de carbón, asbesto o sílice, aumenta la producción de especies reactivas de oxígeno y 8-hidroxiguanisina, lo cual resulta en mutaciones en el ADN y en la activación de los oncogenes. En este contexto, enzimas antioxidantes como la glutatión S-transferasa (GST) (8-10) y las enzimas de reparación por escisión de bases (BER) (OGG1, XRCC1 y APE1) son importantes contra el estrés oxidativo. Se ha encontrado, asimismo, que los polimorfismos de la GST tienen relación con la enfermedad pulmonar intersticial (11), y los de las enzimas OGG1, XRCC1 y APE1 se han asociado con el cáncer pulmonar (12). 
En algunos estudios se han reportado los efectos en la salud y los factores asociados con dicha exposición en trabajadores de minas de carbón; por ejemplo, Wang, et al., evaluaron en el 2013 a 6.373 trabajadores de Estados Unidos y encontraron que el $0,71 \%$ presentaba fibrosis pulmonar masiva relacionada con el deterioro de la función pulmonar registrada en la espirometría, con datos anormales en $13,1 \%$ de ellos, $6,4 \%$ de los cuales presentaba un patrón restrictivo, $5,4 \%$, un patrón obstructivo, y $1,3 \%$, un patrón mixto (13).

En una revisión sistemática del 2014, Mo, et al., revisaron 2.416 estudios, de los cuales 11 evidenciaron que los años de exposición al polvo de carbón, el tipo de trabajo realizado en las minas y el tipo de polvo de carbón, eran factores determinantes de la prevalencia de la neumoconiosis (14). Esta varió según la duración de la exposición: $13,46 \%\left(\mathrm{IC}_{95 \%}: 1,87-33,12\right)$, para exposiciones de 20 años o más; 4,32 \% $\left(\mathrm{IC}_{95 \%}\right.$ : 0,29-12,68\%), para aquellas de 10 a 20 años, y $2,27 \%\left({ }_{1} C_{95 \%}: 0,06-9,20 \%\right)$, para las de menos de 10 años. En otro estudio de neumoconiosis y fibrosis pulmonar masiva en 6.658 mineros de 416 minas en 15 estados de los Estados Unidos, entre 2005 y 2009, se estableció que la prevalencia de neumoconiosis entre los trabajadores de minas subterráneas de carbón variaba entre el 4,8 y el $9 \%$ (15).

En 1995, Rendón, et al., estimaron una prevalencia de neumoconiosis de 5,12\% (IC $\left.{ }_{95 \%} 2,6-7,64\right)$ en Amagá, Antioquia, lo cual contrastaba con los datos encontrados en 1988 , de $1,9 \%$ (16), en tanto que, en el 2000, Orduz, et al., encontraron 189 casos de neumoconiosis en mineros de carbón en el mismo departamento (17). Según el informe sobre enfermedades profesionales en Colombia para el periodo de 2003 a 2005 y un estudio sobre el diagnóstico de la enfermedad en el 2005, en ese año, las enfermedades pulmonares reportadas por las entidades promotoras de salud (EPS), así como los casos de asma y de neumoconiosis, representaron de 3 a $4 \%$ del total de las enfermedades laborales (18).

Sin embargo, en el país no se cuenta con datos actualizados de la prevalencia de neumoconiosis que permitan dimensionar la magnitud del problema y los factores asociados, específicamente las alteraciones espirométricas, ni tampoco hay resultados de análisis genéticos de identificación de los polimorfismos de la glutatión S-transferasa (GST) y las enzimas de reparación OGG1 y XRCC1.
En este contexto, en este estudio se propuso establecer una línea de base de los niveles de polvo de carbón en minas subterráneas, estimar la prevalencia de la neumoconiosis y establecer los factores de riesgo asociados.

\section{Materiales y métodos}

\section{Diseño del estudio}

Se hizo un estudio de corte transversal analítico para estimar la prevalencia de la neumoconiosis y sus factores asociados en mineros de carbón de los departamentos colombianos de Boyacá, Cundinamarca y Norte de Santander.

\section{Población y muestra}

La muestra incluyó 603 empresas del sector minero afiliadas a una administradora de riesgos laborales (ARL), ubicadas en los departamentos objeto del estudio, con un total de 18.174 trabajadores. La muestra fue probabilística, estratificada, aleatoria, con asignación proporcional, bietápica de conglomerados y sin reemplazo. La estratificación se hizo por departamentos (Boyacá, Cundinamarca y Norte de Santander) en dos etapas. En la primera, las unidades primarias de muestreo fueron las empresas de minería subterránea y, en la segunda, las unidades secundarias fueron los trabajadores de las empresas seleccionadas aleatoriamente. La selección de las empresas y de los trabajadores se hizo por muestreo aleatorio simple, con asignación proporcional dentro de cada estrato. En caso de que el trabajador no aceptara participar o no pudiera por no encontrarse en la mina, se reemplazaba de forma aleatoria por otro, teniendo en cuenta los grupos de exposición similar.

El tamaño muestral fue de 476 trabajadores seleccionados aleatoriamente de las 31 empresas escogidas también aleatoriamente en los tres departamentos (18 en Boyacá, 11 en Cundinamarca y 2 en Norte de Santander), con una precisión del $5 \%$ y una confiabilidad del $95 \%$ para una prevalencia esperada alta, del $30 \%$, y un efecto del diseño para conglomerados de 1,5 .

A partir de un listado de todos los trabajadores, se seleccionó un grupo para que respondiera a los cuestionarios estructurados y para tomar las muestras necesarias para los exámenes.

Los criterios de inclusión de los trabajadores fueron los siguientes: decisión voluntaria de participar en el estudio, antigüedad laboral en el sector minero mayor o igual a 10 años y dedicación a trabajos de 
minería subterránea. Se excluyeron las personas que hubieran recibido transfusión sanguínea en los tres meses previos, a las mujeres embarazadas, a personas que hubieran recibido tratamiento con medicamentos antitumorales o radioterapia en los tres meses anteriores, que presentaran alguna condición que contraindicara la espirometría forzada, y aquellos con enfermedad respiratoria activa o en tratamiento.

\section{Instrumentos y procedimientos para la recolección de la información}

Se utilizaron tres cuestionarios. En el primero, dirigido a los trabajadores, se recolectó la información sobre las características sociales y demográficas (edad, sexo, escolaridad, ubicación de la vivienda, estrato socioeconómico, nivel educativo, estado civil), ocupacionales (antigüedad en el desempeño de actividades de minería, cargos desempeñados, oficio y tiempo de exposición en el momento de responder al cuestionario), toxicológicas (condición de fumador y consumo de alcohol) y lo relacionado con los síntomas respiratorios. El segundo cuestionario era la historia clínica con la información de la evaluación médica ocupacional, elaborada por médicos especialistas con licencia vigente de salud ocupacional. Con el tercer cuestionario se evaluó el ambiente laboral mediante preguntas dirigidas a la empresa a cargo de un higienista del equipo de investigación, quien inspeccionó los ambientes laborales para caracterizar la exposición y establecer los grupos de exposición similar.

Los exámenes practicados a los trabajadores incluyeron una radiografía de tórax tomada y leída según los criterios exigidos por la guía técnica de la Organización Internacional del Trabajo (OIT), una espirometría forzada realizada por una terapeuta respiratoria, y el análisis genético para identificar los polimorfismos de la glutatión S-transferasa y de las enzimas de reparación OGG1 y XRCC1.

La lectura de la radiografía de tórax estuvo a cargo de dos lectores B certificados por el National Institute for Occupational Safety and Health (NIOSH) de los Estados Unidos.

La espirometría, la cual se considera como una herramienta práctica en el seguimiento de los trabajadores, pues permite evaluar cuantitativamente las características mecánicas de la respiración, se aplicó con base en los estándares de la American Thoracic Society (ATS) y de la European Respiratory Society (ERS), cuyos valores se consideran normales cuando la CVF es igual o mayor al $80 \%$ de lo esperado según la talla, el peso, la edad y el sexo, cuando el $\mathrm{VEF}_{1}$ es igual o mayor de $80 \%$ de lo esperado, y cuando la relación entre una y otro es igual o mayor de 70 $\%$ (19).

Para el análisis genético se tomó una muestra de sangre periférica en dos tubos con EDTA, los cuales se almacenaron a $4{ }^{\circ} \mathrm{C}$ y se transportaron al laboratorio dentro de las 48 horas siguientes a la toma de la muestra para la extracción del ADN mediante un estuche comercial. Para determinar los polimorfismos de los genes GSTM1 y GSTT1, se empleó una reacción en cadena de la polimerasa múltiple (PCR múltiplex). Los productos amplificados se verificaron por electroforesis en geles de agarosa al $2 \%$, y los genotipos del GSTM1 y el GSTT1 se determinaron mediante la detección de bandas de 215 pb y 459 pb, respectivamente. El genotipo nulo se definió como la ausencia de producto en la PCR.

Para el gen de reparación OGG1, se hizo una PCR con la enzima de restricción Stal y los productos se visualizaron en gel de agarosa al $3 \%$. El heterocigoto Ser/Cys $(\mathrm{C} / \mathrm{G})$ tiene tres bandas de 100, 107 y 207 pb; el homocigoto Ser/Ser (C/C), una sola banda de $207 \mathrm{pb}$, porque no se corta con la enzima, y el homocigoto Cys/Cys (G/G) tiene dos bandas de 100 y $107 \mathrm{pb}$.

Se analizaron dos polimorfismos del gen de reparación XRCC1, uno en el codón 194, localizado en el exón 6 , y el segundo, en el codón 399, ubicado en el exón 10. Las muestras de ADN se amplificaron mediante PCR múltiple. Los fragmentos amplificados fueron digeridos con la enzima de restricción Mspl para determinar los polimorfismos correspondientes de los dos codones.

Una vez definidos y validados los grupos de exposición similar, se midió la concentración en aire de la fracción respirable de polvo de carbón y sílice, para lo cual se seleccionó aleatoriamente a los trabajadores y se evaluaron sus ambientes personales mediante el método de análisis gravimétrico del NIOSH para personas (0600) y ambientes (7602). La concentración de carbón se midió con el método validado, el cual incluye la recolección de la muestra en filtros de PVC, utilizando un ciclón de nylon de $10 \mathrm{~mm}$ de orificio y el análisis gravimétrico. El límite de detección de la técnica fue de 0,03 mg. Para el polvo de sílice, se 
utilizó un espectrofotómetro de absorción de rayos infrarrojos y se determinó la fracción contenida en la muestra retenida en los filtros. Se recolectaron 476 muestras y se enviaron a un laboratorio de higiene y toxicología industrial para su análisis.

El valor umbral de referencia se estableció según los parámetros establecidos por la American Conference of Governmental Industrial Hygienists (ACGIH), en tanto que, para el promedio ponderado en el tiempo, se tuvo en cuenta que la jornada laboral en las empresas evaluadas era de 48 horas semanales, y se ajustó empleando el modelo de Brief y Scala contenido en el libro "Patty's Industrial Hygiene and Toxicology", recomendado por la ACGIH. Dado que los valores límites permisibles están establecidos para jornadas laborales de 40 horas semanales, los valores usados en el presente estudio se ajustaron así: para polvo de carbón bituminoso, a $0,7 \mathrm{mg} / \mathrm{m}^{3}$, y para el de sílice cristalina, a $0,020 \mathrm{mg} / \mathrm{m}^{3}$.

Para determinar el riesgo de exposición a contaminantes químicos, se calculó el índice de riesgo, definido como el cociente entre la concentración evaluada en campo y el valor límite permisible corregido. Con base en ello, se establecieron las siguientes escalas de niveles de riesgo: para el polvo de carbón bituminoso, el índice de riesgo se consideró bajo (menos de 0,5), medio (de 0,5 a menos de 1), alto (de 1 a menos de 5) o grave (de 5 o mayor), para el polvo de sílice cristalina, el valor se consideró bajo (menos de 0,5), medio (de 0,5 a menos de 1), alto (de 1 a menos de 2), grave (de 2 a menos de 5), o crítico (de 5 o mayor).

\section{Operacionalización de las variables}

Las principales variables independientes fueron la exposición al polvo de carbón y de sílice en minería subterránea (concentración en aire de la fracción respirable de polvo de carbón y de sílice), la antigüedad en el oficio de la minería subterránea (meses laborados en el oficio), los polimorfismos genéticos (los polimorfismos GSTM1 y GSTT1 de la glutatión S-transferasa y las enzimas de reparación OGG1 y XRCC1), las alteraciones espirométricas y los valores de la espirometría (según los estándares de la ATS y la ERS).

Otras variables independientes fueron: el departamento (lugar de ubicación de la empresa), el tamaño de la empresa (pequeñas: de 1 a 50 trabajadores, medianas: de 51 a 99 trabajadores, y grandes: de más de 100 trabajadores), los grupos homogéneos de exposición similar a polvo de carbón y de sílice, y el tiempo en el cargo actual (en meses).

Las variables de confusión fueron: la edad (años cumplidos), los antecedentes de tabaquismo (tiempo total en meses como fumador) y la enfermedad respiratoria (antecedentes reportados por los trabajadores durante su vida), en tanto que la variable dependiente fue la neumoconiosis (trabajadores con opacidades u otras alteraciones en la radiografía de tórax indicativas de neumoconiosis).

\section{Prueba piloto y estrategias de control de la calidad de los datos}

Se hizo una prueba piloto en tres empresas de minas subterráneas, con el fin de hacer los ajustes necesarios de los instrumentos, los tiempos y los movimientos, y estandarizar todos los procedimientos. Las empresas participantes en el estudio piloto no formaron parte de la muestra seleccionada para el estudio definitivo.

La recolección de la información estuvo a cargo de profesionales del equipo de investigación, quienes usaron los cuestionarios estructurados de manera individual con cada uno de los participantes seleccionados de la muestra. Antes de iniciar la fase de recolección de la información, se capacitó a los profesionales encargados para garantizar su uso estandarizado. Las encuestas y los exámenes de laboratorio se hicieron en un mismo día con cada trabajador.

Para garantizar la calidad de la información recolectada en los cuestionarios, al finalizar la jornada diaria un profesional del equipo de investigadores la revisaba para establecer si había inconsistencias o datos faltantes que pudieran afectar su análisis. En caso de que así fuera, se verificaba nuevamente la información con la empresa 0 el trabajador participante. Asimismo, esta se revisaba antes de ser incluida y analizada en la base de datos del proyecto.

Para sistematizar la información se diseñó una base de datos en el módulo de "vista de variables" y posteriormente se digitó en "vista de datos" del programa SPSS Statistics ${ }^{\circledR}$, versión 25.0. Los datos digitados se verificaron con el control automático de este mismo programa.

\section{Análisis estadístico}

El diseño de los estimadores y pruebas estadísticas fue probabilístico, estratificado y multietápico por conglomerados, según el módulo de muestras 
probabilísticas complejas del SPSS Statistics ${ }^{\circledR}$, versión 25.0.

Se estimó la prevalencia de la neumoconiosis con su respectivo intervalo de confianza del $95 \%$; la precisión de los estimadores se midió con el error estándar (EE) y el error estándar relativo (EER), teniendo en cuenta el criterio de calidad de los estimadores de la National Statistical Agency (1985) de Canadá para el EER (calidad A: 0-15\%, B: $15,1-30 \%$, C: $30,1-50 \%$, y D: $>50 \%$ ) .

Las asociaciones entre las variables cualitativas independientes y la neumoconiosis se evaluaron con la prueba de asociación de razón de verosimilitud, la razón de prevalencias $(\mathrm{RP})$ y su respectivo intervalo de confianza del $95 \%$. Para el análisis multivariado de la neumoconiosis, se empleó un modelo de regresión de Cox con tiempo constante de riesgo y estimadores sólidos de varianza para muestreos estratificados multietápicos por conglomerados. Previamente, se evaluó la multicolinealidad de los factores con pruebas de correlación, y se establecieron el grado y el tipo de confusión (negativa, positiva o cualitativa). El modelo final se seleccionó con el método de tipo jerárquico (20). El nivel de significación utilizado en las pruebas estadísticas, fue de $5 \%(p<0,05)$.

\section{Consideraciones éticas}

El estudio obtuvo la aprobación de los comités técnico y de ética del Instituto Nacional de Salud y de la Universidad del Rosario, y se ajustó a las consideraciones éticas establecidas en la Declaración de Helsinki y la Resolución 8430 de 1993 del Ministerio de Salud de la República de Colombia, según la cual se consideró como de riesgo mínimo para los participantes.

Antes de iniciar la recolección de la información, los investigadores informaron a todos los participantes sobre el tipo de estudio, su importancia, los beneficios, los riesgos y el tipo de muestras que se tomarían, y posteriormente, se procedió a la firma del consentimiento informado.

\section{Resultados}

En la muestra probabilística se incluyeron 476 trabajadores de tres departamentos (232 de Boyacá, 215 de Cundinamarca y 29 de Norte de Santander) de 31 empresas de minería subterránea (18 en Boyacá, 11 en Cundinamarca y 2 en Norte de Santander). La distribución de los trabajadores según el tamaño de las empresas, fue la siguiente: en empresas pequeñas, $53,6 \% \quad(n=255)$; en medianas, $28,4 \%(n=135)$, y en grandes, $18,1 \%$ $(n=86)$.

\section{Características demográficas y ocupacionales}

La edad de los trabajadores variaba entre los 20 y los 76 años, con un promedio de 43,0 $\pm 10,0$ años; la mayoría eran hombres (solo se encontró una mujer laborando en una de las minas). Las edades de los trabajadores fluctuaban entre los 35 y los 49,9 años, su estrato socioeconómico correspondía al 2 , su nivel educativo era la primaria y residían en zonas rurales (cuadro 1).

El promedio de antigüedad en el trabajo en las minas fue de 19,3 $\pm 9,1$ años (mediana de 16 años), y $30,5 \%$ de los trabajadores tenía 25 años o más de antigüedad. El tiempo en el cargo del trabajador en el momento del estudio variaba entre un mes y 34 años, con un promedio de $5,4 \pm 6,1$ años y una mediana de tres años.

Los cargos se clasificaron con base en los cinco grupos de exposición similar: frente de explotación y avance de túnel, transporte, mantenimiento, servicios, y punto de carga interno en la mina. De estos, el más frecuente (más del $80,0 \%$ ) fue el de explotación y avance de túnel; en este grupo, el cargo de mayor frecuencia $(74,6 \%)$ fue el de picador; en el área de transporte, el más frecuente $(90,0 \%)$ fue el de 'malacatero' y, en la de punto de carga interna en la mina, el más frecuente $(57,9 \%)$ fue el de reforzador.

En cuanto a los antecedentes toxicológicos, el $43,5 \%$ de los trabajadores $(n=207)$ reportó haber fumado alguna vez en la vida y el 16,0\% $(n=76)$ seguía haciéndolo en el momento del estudio, con un tiempo promedio como fumadores de $13 \pm 10,6$ años (rango de 1 a 48); 5,5\% $(n=26)$ de estos fumaba en el sitio de trabajo un promedio de $3,8 \pm 4,7$ cigarrillos por día (rango de 1 a 30 ).

\section{Exposición al polvo de carbón y de sílice}

Según los niveles de exposición al polvo de carbón, los de riesgo alto $(57,6 \%)$ y grave $(30,7$ $\%)$ fueron los más frecuentes seguidos por el nivel medio $(5,0 \%)$ y el bajo $(5,4 \%)$. Los niveles más frecuentes de exposición al polvo de sílice fueron el grave $(44,6 \%)$ y el crítico $(35,2 \%)$, seguidos del alto $(13,6 \%)$ y el medio $(6,5 \%)$.

\section{Evaluación de la espirometría}

Los resultados de la espirometría en los trabajadores se clasificaron así: en el patrón normal estaba el $90,1 \%$ de ellos; en el patrón obstructivo, el 3,1 \%; 
Cuadro 1. Características demográficas de los trabajadores de minas subterráneas en los tres departamentos (Boyacá, Cundinamarca, Norte de Santander)

\begin{tabular}{llcc}
\hline \multicolumn{1}{c}{ Caracteristicas } & $\mathbf{n}$ & $\%$ \\
\hline Grupos etarios & $20-34,9$ & 112 & 23,6 \\
(años) & $35-49,9$ & 229 & 48,2 \\
& $\geq 50$ & 134 & 28,2 \\
& Total & 475 & 100,0 \\
Sexo & Masculino & 475 & 99,8 \\
Estrato & Femenino & 1 & 0,2 \\
socioeconómico & 1 & 82 & 17,4 \\
& 2 & 252 & 53,4 \\
& 3 & 120 & 25,4 \\
& 4 & 15 & 3,2 \\
Nivel educativo & Ninguno & 3 & 0,6 \\
& Primaria & 9 & 1,9 \\
& Secundaria & 347 & 72,9 \\
& Técnica & 6 & 23,5 \\
Ubivienda & Universitaria & 2 & 1,3 \\
& Rural & 279 & 58,6 \\
& & 197 & 41,4 \\
\hline
\end{tabular}

en el restrictivo, el 1,2\%, y en el mixto, el 0,6\%. El promedio más el error estándar de la relación entre el volumen espiratorio forzado en 1 segundo $\left(\mathrm{VEF}_{1}\right)$ y la capacidad vital forzada (CVF) fue de $81,64 \pm 1,25 \%$ (rango de 58,0 a $124,0 \%$ ), y el del flujo espiratorio forzado de 25 a $75 \%$ (FEF 25-75) fue de $87,9 \pm 2,14 \%$ (rango de 33,0 a $199,0 \%$ ).

\section{Polimorfismos genéticos}

En el $66,9 \%$ los trabajadores, se encontró un genotipo normal de GSTM1 y el 33,1\% tuvo un genotipo nulo; en cuanto a la enzima GSTT1, el genotipo fue normal en el $83,2 \%$ y nulo en el $16,8 \%$.

\section{Prevalencia de neumoconiosis}

La prevalencia estimada de neumoconiosis según los criterios de la OIT para trabajadores de minas subterráneas, fue de 33,8 \% ( $\left(\mathrm{IC}_{95 \%} 27,0-41,3 \%\right)$.

\section{Factores asociados con la neumoconiosis}

Hubo diferencias significativas entre los departamentos en cuanto a la presencia de neumoconiosis, siendo mayor en Cundinamarca, seguida por Boyacá y, con menor prevalencia, por Norte de Santander; en las categorías de riesgo de exposición al polvo de carbón y de sílice, no se encontró una asociación significativa, como tampoco la hubo con el cargo ocupado por el trabajador. En valores numéricos, el promedio de la escala de polvo de carbón fue significativamente mayor en los trabajadores con neumoconiosis que en quienes no la presentaban $(5,44 \mathrm{Vs}$. $4,40 ; p=0,038)$; en cuanto a la de sílice, no hubo diferencias $(p=0,158)$.

Se registró una tendencia lineal significativa en la asociación entre la neumoconiosis, el aumento del tiempo de antigüedad laboral y el cargo ocupado en el momento del estudio, así como el aumento de la edad del trabajador. También se registró una asociación significativa con el tamaño de la empresa, siendo mayor la prevalencia de la neumoconiosis en las empresas grandes $y$ medianas.

Los resultados de las alteraciones de la espirometría no se asociaron significativamente con la neumoconiosis. Se encontraron diferencias entre los trabajadores con neumoconiosis y sin ella en los valores de la relación entre el $\mathrm{VEF}_{1}$ y la CVF en la espirometría $(79,85$ Vs. 82,53; $\mathrm{p}=0,044)$ y el FEF $25-75(84,04 \mathrm{Vs} .89,81 ; \mathrm{p}=0,30)$. Los antecedentes de tabaquismo $(p=0,353)$ y de enfermedad respiratoria $(p=0,404)$ no se asociaron con la neumoconiosis (cuadro 2).

\section{Análisis multivariado}

El modelo de regresión multivariada de Cox, con tiempo de riesgo constante para la neumoconiosis, se elaboró teniendo en cuenta las variables con un $p<0,20$ en el análisis bivariado, en tanto que en el modelo final se encontró asociado significativamente con el trabajo en empresas medianas comparado con empresas pequeñas $\left(\mathrm{RP}=2,00 ; \quad \mathrm{IC}_{95 \%}\right.$ 0,995-2,690; $\left.\mathrm{p}=0,052\right)$, con el nivel de exposición grave al polvo de carbón en comparación con el nivel bajo ( $\mathrm{RP}=2,055 ; \mathrm{IC}_{95 \%}$ $1,043-4,048 ; p=0,038$ ) y con una antigüedad en la minería subterránea de 25 años o más comparada con menos de 25 años (para 25,0-29,9 años: $\mathrm{RP}=2,199 ; \mathrm{IC}_{95 \%} 1,449-3,338 ; \mathrm{p}=0,001 ; \mathrm{y}$ para 30 años o más: $R P=2,072 ; \quad I_{95 \%} 1,321-3,250$; $\mathrm{p}=0.003)$.

\section{Discusión}

De los 476 trabajadores valorados en los tres departamentos, se detectaron 165 casos con características radiológicas correspondientes a neumoconiosis según los criterios de la OIT, es 
Cuadro 2. Estimación de la prevalencia de neumoconiosis y errores de muestreo según los factores asociados

\begin{tabular}{|c|c|c|c|c|c|c|c|}
\hline \multirow[t]{2}{*}{ Variable } & \multirow{2}{*}{$\begin{array}{c}\text { Prevalencia } \\
\%\end{array}$} & \multirow{2}{*}{$\begin{array}{c}\text { Error } \\
\text { estándar }\end{array}$} & \multirow{2}{*}{$\begin{array}{c}\text { Error } \\
\text { estándar } \\
\text { relativo }\end{array}$} & \multicolumn{3}{|c|}{$I_{95 \%}$} & \multirow[t]{2}{*}{ Significación } \\
\hline & & & & $\begin{array}{l}\text { Límite } \\
\text { inferior }\end{array}$ & $\begin{array}{c}\text { Límite } \\
\text { superior }\end{array}$ & $\begin{array}{l}\text { Efecto del } \\
\text { diseño }\end{array}$ & \\
\hline \multicolumn{4}{|l|}{ Departamento } & & & & 0,044 \\
\hline Boyacá & 29,9 & 5,4 & 18,1 & 20,0 & 42,0 & 3,86 & \\
\hline Cundinamarca & 42,3 & 3,9 & 9,3 & 34,5 & 50,5 & 1,15 & \\
\hline Norte Santander & 17,2 & 6,4 & 37,2 & 7,7 & 34,3 & 0,84 & \\
\hline \multicolumn{4}{|c|}{ Nivel de exposición a carbón } & & & & 0,427 \\
\hline Bajo & 20,3 & 7,2 & 35,6 & 9,3 & 39,0 & 0,88 & \\
\hline Medio & 38,9 & 16,6 & 42,6 & 13,3 & 72,7 & 2,50 & \\
\hline Alto & 32,3 & 5,4 & 22,4 & 44,2 & 16,7 & 3,27 & \\
\hline \multicolumn{4}{|c|}{ Nivel de exposición a sílice } & & & & 0,168 \\
\hline Medio & 39,7 & 12,3 & 30,9 & 18,7 & 65,3 & 1,76 & \\
\hline Alto & 21,5 & 5,2 & 24,4 & 12,7 & 34,1 & 0,95 & \\
\hline Grave & 31,1 & 6,2 & 20,1 & 19,9 & 45,1 & 3,46 & \\
\hline Crítico & 41,0 & 4,1 & 9,9 & 33,1 & 49,5 & 1,02 & \\
\hline \multicolumn{4}{|c|}{ Antigüedad laboral (años) } & & & & $<0,001$ \\
\hline$<15$ & 17,9 & 3,8 & 21,3 & 11,3 & 27,0 & 2,11 & \\
\hline $15-19,9$ & 33,2 & 8,2 & 24,6 & 18,9 & 51,5 & 2,04 & \\
\hline $20-24,9$ & 43,7 & 6,6 & 15,1 & 30,9 & 57,3 & 1,15 & \\
\hline $25-29,9$ & 53,3 & 7,3 & 13,7 & 38,5 & 67,5 & 1,21 & \\
\hline$\geq 30$ & 54,3 & 5,1 & 9,3 & 43,9 & 64,3 & 0,85 & \\
\hline \multicolumn{2}{|l|}{ Grupos etarios } & & & & & & $<0,001$ \\
\hline $20-34,9$ & 11,1 & 3,2 & 29,2 & 6,0 & 19,7 & 1,29 & \\
\hline $35-49,9$ & 33,0 & 4,1 & 12,5 & 25,2 & 41,9 & 1,81 & \\
\hline$\geq 50$ & 55,8 & 4,5 & 8,1 & 46,4 & 64,8 & 1,08 & \\
\hline \multicolumn{4}{|c|}{ Tiempo en el cargo actual (meses) } & & & & 0,014 \\
\hline$<2$ & 26,4 & 4,9 & 18,7 & 17,6 & 37,7 & 2,08 & \\
\hline $2-5$ & 31,7 & 4,0 & 12,7 & 24,1 & 40,5 & 1,29 & \\
\hline$>5$ & 44,2 & 4,7 & 10,7 & 34,9 & 54,0 & 1,17 & \\
\hline \multicolumn{3}{|l|}{ Cargo GES } & & & & & 0,241 \\
\hline $\begin{array}{l}\text { Explotación y } \\
\text { avance en túnel }\end{array}$ & 35,1 & 3,8 & 10,8 & 27,8 & 43,2 & 2,56 & \\
\hline Transporte & 18,4 & 7,3 & 40,0 & 7,6 & 38,0 & 1,13 & \\
\hline Punto de carga & 39,0 & 9,6 & 24,7 & 21,8 & 59,4 & 0,663 & \\
\hline Mantenimiento & 26,6 & 10,2 & 38,4 & 11,0 & 51,5 & 1,58 & \\
\hline Servicios & 46,8 & 13,2 & 28,1 & 23,0 & 72,2 & 0,38 & \\
\hline \multicolumn{3}{|c|}{ Tamaño de la empresa } & & & & & 0,002 \\
\hline Grande & 40,2 & 2,1 & 15,8 & 36,0 & 44,5 & 0,16 & \\
\hline Mediana & 49,0 & 3,0 & 6,0 & 42,9 & 55,0 & 0,43 & \\
\hline Pequeña & 25,1 & 5,2 & 20,8 & 16,0 & 37,2 & 4,04 & \\
\hline \multicolumn{2}{|l|}{ Espirometría } & & & & & & 0,202 \\
\hline Alteraciones & 43,6 & 8,4 & 19,3 & 27,7 & 60,9 & 1,39 & \\
\hline Normal & 32,9 & 3,4 & 10,5 & 26,2 & 40,3 & 2,33 & \\
\hline \multicolumn{2}{|l|}{ GSTT1 } & & & & & & 0,316 \\
\hline Positivo & 32,8 & 3,5 & 10,7 & 26,1 & 40,4 & 2,26 & \\
\hline Negativo & 39,1 & 6,7 & 17,1 & 26,5 & 53,3 & 1,54 & \\
\hline \multicolumn{2}{|l|}{ GSTM1 } & & & & & & 0,339 \\
\hline Positivo & 32,5 & 3,8 & 11,7 & 25,2 & 40,7 & 2,14 & \\
\hline Negativo & 36,7 & 4,5 & 12,4 & 27,9 & 46,4 & 1,43 & \\
\hline \multicolumn{2}{|l|}{ OGG1 } & & & & & & 0,246 \\
\hline Ser/Ser & 31,3 & 3,9 & 12,6 & 23,9 & 39,9 & 2,05 & \\
\hline Ser/Cys & 37,4 & 4,3 & 11,6 & 29,0 & 46,6 & 1,53 & \\
\hline Cys/Cys & 39,3 & 10,4 & 26,4 & 21,0 & 61,2 & 0,43 & \\
\hline OGG1 & & & & & & & 0,145 \\
\hline Positivo & 31,4 & 3,9 & 12,5 & 24,0 & 40,0 & 2,03 & \\
\hline Negativo & 37,3 & 4,1 & 10,9 & 29,4 & 46,0 & 1,43 & \\
\hline
\end{tabular}

Error estándar relativo >50 \% GES: grupos de exposición similar 
decir, una prevalencia de 33,8 \%, más alta que la registrada en estudios previos en Colombia (5\%), en Estados Unidos (4\%) y en China (6\%) $(13,14,16,17)$.

La prevalencia de la neumoconiosis fue mayor en los trabajadores con edades por encima de los 50 años, lo que coincide con otros estudios (16). Sin embargo, la edad promedio (42,9 años) fue inferior a la reportada en Estados Unidos (52,6 años) y la República Checa $(49,7$ años) $(21,22)$. Además, en el presente estudio sobresale el hecho de que el extremo inferior de edad fue de 20 años, dato que podría explicarse por el inicio de labores directas o indirectas en los socavones a muy temprana edad.

En cuanto al nivel de educación, la prevalencia de neumoconiosis fue mayor en trabajadores con un nivel educativo bajo; debe recordarse que la mayoría de los trabajadores tenía un nivel educativo de primaria, inferior al promedio nacional para mayores de 15 años, que es de 9,41 años de educación (23). La prevalencia se asoció, asimismo, con más de 25 años de experiencia, lo que coincide con los datos de Estados Unidos entre el 2005 y el 2009 (24).

En la revisión sistemática de Mo, et al., se evidenció que los años de exposición al polvo de carbón era uno de los factores de los cuales dependía la prevalencia de la neumoconiosis. Aunque el diagnóstico de la enfermedad se basa fundamentalmente en los antecedentes de exposición, los cuales incluyen los años laborados, las manifestaciones clínicas y los hallazgos radiológicos interpretados según los criterios de la OIT, es importante anotar que, en algunos casos, los hallazgos radiológicos no son coherentes con los síntomas o con la historia de exposición. En estos casos, se requieren otras técnicas diagnósticas, como la histopatología o las imágenes. La primera, aunque sea confirmatoria, no es la más recomendada por su carácter invasivo, por lo que se prefieren las segundas, especialmente la tomografía computadorizada (4).

En cuanto al cargo ocupado por el trabajador, la prevalencia de neumoconiosis fue del $35,1 \%$ en aquellos cargos relacionados con la explotación y el avance de la mina (picadores, 'tecleros' y fronteros), los cuales son de mayor riesgo por la acción directa de picar y remover la tierra del tajo de carbón. Sin embargo, a diferencia de otro estudio previo, en este no se encontró una diferencia significativa (25). Además, tampoco se encontró una asociación entre la neumoconiosis y el tabaquismo, contrario a lo descrito en otro estudio (26).
La mayoría de los mineros trabajaba en minas pequeñas (menos de 50 trabajadores), mecanizadas o mixtas, y en ambientes con niveles de riesgo entre graves y críticos por la exposición al polvo de sílice y de carbón. Se encontró una asociación entre el nivel grave de exposición al polvo de carbón y la presencia de neumoconiosis, como se ha podido demostrar en otros estudios (27).

En Estados Unidos, se han registrado tasas de enfermedad mayores entre los mineros de minas pequeñas (menos de 155 mineros) (28), información que no es comparable con la de este estudio debido a las diferencias en la clasificación según el tamaño de las empresas.

Los valores de $\mathrm{VEF}_{1} / \mathrm{CVF}$ y el FEF 25-75 fueron significativamente menores en los trabajadores con neumoconiosis, a diferencia de lo registrado en otros estudios, en los cuales la disminución de la CVF y el VEF 1 ha sido mayor $(13,29)$. Las alteraciones obstructivas detectadas en la espirometría fueron frecuentes, lo cual hace pensar que la bronquitis crónica y la enfermedad pulmonar obstructiva crónica (EPOC) también podrían estar presentes en esta población. Por otro lado, no se encontró el polimorfismo genético de la GST como un factor de riesgo para la neumoconiosis. A diferencia de los otros estudios llevados a cabo en el país, en este se determinaron la prevalencia de la neumoconiosis y los factores asociados empleando una muestra probabilística $(16,17)$. Según los datos del censo nacional minero por departamentos, realizado por el Ministerio de Minas y Energía en el 2012, en Boyacá el 69,0 \% de las unidades de producción minera de carbón poseían título minero, en Cundinamarca, el 50,8\%, y en Norte de Santander, el 64,0\%. Las empresas incluidas en el estudio tenían título minero y estaban afiliadas a una administradora de riesgos laborales. Es posible que en las que no estaban afiliadas, la prevalencia de neumoconiosis fuera mayor, ya que en ellas las condiciones de seguridad y salud en el trabajo serían más desfavorables. En este estudio se evaluaron las medidas de higiene y seguridad industrial en cada una de las empresas incluidas y, aunque dicha información no se presenta en este artículo, es importante mencionar que los resultados solo pueden ser extrapolables a empresas afiliadas a administradoras de riesgos laborales (30).

Este estudio fue de corte transversal, por lo que la exposición de los trabajadores al polvo 
de carbón y de sílice se midió en el momento en que se llevó a cabo el trabajo de campo y no representa la exposición acumulada, para lo cual se requeriría de estudios de cohorte que permitieran observar la exposición en el tiempo. La recolección de la información, al igual que todos los exámenes, se hizo en un mismo día para cada trabajador. La lectura de las placas radiográficas solo se llevó a cabo días después, por lo que los médicos que hicieron las evaluaciones no conocían el diagnóstico de las lecturas en el momento del examen, con lo cual la calidad de la información recolectada y de los resultados no se vio afectada.

En cuanto al control de los sesgos de confusión, los análisis de la estimación de la asociación entre la exposición ocupacional al polvo de carbón y los factores de riesgo, se ajustó según las variables de la edad, la antigüedad laboral y el hábito del tabaquismo. Por otro lado, con el objeto de minimizar los sesgos de memoria durante el suministro de la información requerida, no se utilizaron preguntas abiertas sobre los antecedentes personales incluidos en la historia médica.

Un posible sesgo de selección podría haberse registrado si se considera que la neumoconiosis es una enfermedad de desarrollo crónico, por lo que sus manifestaciones clínicas y los cambios radiológicos aparecen, por lo general, a partir de los 10 años de exposición. Sin embargo, en este estudio se seleccionaron trabajadores que tenían ese tiempo mínimo de exposición, en tanto que en otros estudios se ha evaluado a toda la población de mineros (4).

Por otra parte, en próximos estudios deberán incluirse personas más jóvenes, con el fin de detectar entre ellas signos precoces de la enfermedad o de compromiso pulmonar.

En Estados Unidos, China y otros países productores de carbón crece el interés por controlar el desarrollo de la neumoconiosis en los trabajadores expuestos $(14,31)$, pero la alta prevalencia sugiere que las medidas propuestas hasta el momento no han sido suficientes. Además, en los últimos años se ha podido establecer que un factor determinante en el aumento de la prevalencia es la generación de grandes volúmenes de carbón debido al empleo de tecnología de punta (32).

Deben implementarse medidas sistemáticas de prevención poblacional y ambiental, pues, a pesar de que los elementos de protección respiratoria y los controles de ingeniería para el control del polvo están estandarizados, los equipos y los procesos implementados pueden variar de una mina a otra (31). Por otro lado, se necesita evaluar, vigilar y hacer el seguimiento de los trabajadores, ya que, dada la prevalencia encontrada, podría haber cerca de 1.400 mineros con sospecha de neumoconiosis todavía no detectados en estos departamentos, lo cual exige una búsqueda activa inmediata.

\section{Recomendaciones}

Este tipo de estudios debe repetirse ampliando el número de trabajadores evaluados, de manera que se conviertan en modelos operativos sistemáticos a nivel nacional. Los servicios médicos locales de las zonas mineras deberán aducir argumentos técnicos válidos para una vigilancia activa de gran calidad y confianza. En este sentido, los ministerios de Salud y de Trabajo cuentan con un estudio que justifica el incremento de las acciones orientadas a la detección temprana de los casos y a un mayor compromiso de disminuir la silicosis.

Los empleadores deben revisar sus responsabilidades frente al control más efectivo del riesgo, pues no pueden mantenerse valores de prevalencia en los niveles detectados. Los métodos de control empleados deberán revisarse, ya que, aparentemente, no son eficaces. Es esencial adelantar una vigilancia más activa y permanente, con énfasis en el control del riesgo, así como la observación permanente de la salud de los trabajadores mediante mejores tecnologías.

Los trabajadores, por su parte, deben incrementar sus acciones de autocuidado para evitar la pérdida de años de vida, y apoyar las actividades de los empleadores para disminuir significativamente el riesgo. Por otra parte, en estudios futuros se debe ampliar la valoración de las alteraciones genéticas para, así, reconocer precozmente las alteraciones de la salud asociadas con este tipo de exposiciones.

Hay un subregistro importante de casos, lo cual hace necesario organizar un "programa obligatorio" de vigilancia epidemiológica a nivel nacional en el campo de la minería en general, y la del carbón, en particular, dada la creciente importancia de esta actividad en la economía nacional y el aumento del número de trabajadores expuestos a material en partículas. Debe señalarse que las recomendaciones en torno al establecimiento de un programa adecuado de vigilancia 
epidemiológica en el ámbito de la salud ambiental y de los trabajadores de la minería subterránea del carbón, las cuales están contenidas en las guías elaboradas por el Ministerio de la Protección Social en el 2006, aún no se han implementado.

\section{Agradecimientos}

A los trabajadores y empresas participantes en el estudio, a la Universidad del Rosario, a la Universidad de los Andes, a Positiva Compañía de Seguros ARL, al Instituto Nacional de Salud y a la Gobernación de Boyacá.

\section{Conflicto de intereses}

Los autores declaran no tener conflicto de intereses.

Financiación

El estudio fue financiado con recursos de la Universidad del Rosario, la Universidad de los Andes, Positiva Compañía de Seguros ARL, el Instituto Nacional de Salud y la Gobernación de Boyacá, y cofinanciado por Colciencias mediante el contrato 379-2011.

\section{Referencias}

1. Fishwick D, Barber C. Pneumoconiosis. Medicine. 2012;40: 310-3. https://doi.org/10.1016/j.mpmed.2012.03.011

2. Unidad de Planeación Minero Energético. Zonas carboníferas de Colombia. Ministerio de Minas y Energía. Bogotá. Fecha de consulta: 6 de abril de 2017 . Disponible en: http:// www.upme.gov.co/guia_ambiental/carbon/areas/zonas/indice. $\mathrm{htm}$

3. Bernales B, Alcalino J, Solís, R. Situación de la exposición laboral a sílice en Chile. Ciencia y Trabajo. 2008;20:11-7.

4. Ministerio de la Protección Social. Guía de atención integral de salud ocupacional basada en la evidencia para neumoconiosis (silicosis, neumoconiosis del minero de carbón y asbestosis). Bogotá, D.C.: Imprenta Nacional de Colombia; 2007. p. 75-93

5. Zhai R, Liu G, Ge X, Yang C, Huang C, Wu C, et al. Genetic polymorphisms of MnSOD, GSTM1, GSTT1, and OGG1 in coal workers' pneumoconiosis. J Occup Environ Med. 2002;44:372-7.

6. Organización Mundial de la Salud. Informe sobre la salud en el mundo 2007: un porvenir más seguro. Protección de la salud pública mundial en el siglo 21. Ginebra: OMS; 2007. p. 7

7. Chong S, Lee KS, Chung MJ, Han J, Kwon OJ, Kim TS. Pneumoconiosis: Comparison of imaging and pathologic findings. Radiographics. 2006;26:59-77. https://doi.org/10.1148/ rg.261055070

8. Yucesoy B, Luster MI. Genetic susceptibility in neumoconiosis. Toxicol Lett. 2007;168:249-54. https://doi. org/10.1016/j.toxlet.2006.10.021
9. Costa C, Pasquale RD, Silvari V, Barbaro M, Catania S. In vitro evaluation of oxidative damage from organic solvent vapours on human skin. Toxicol In vitro. 2006;20:324-31. https://doi.org/10.1016/j.tiv.2005.08.007

10. Heuser V. Evaluation of genetic damage in Brazilian footwear workers. Biomarkers of exposure, effect and suscep tibility. Toxicology. 2007;10:1-13. https://doi.org/10.1016/j.tox.20 07.01.011

11. Borm PJ, Schins RP. Genotype and phenotype in susceptibility to coal workers' pneumoconiosis. The use of cytokines in perspective. Eur Respir J Suppl. 2001;32:12733s.

12. Li Z, Guan W, Li MX, Zhong ZY, Qian CY, Yang XQ, et al. Genetic polymorphism of DNA base-excision repair genes (APE1, OGG1 and XRCC1) and their correlation with risk of lung cancer in a Chinese population. Arch Med Res.2011;42:22634. https://doi.org/10.1016/j.arcmed.2011.04.005

13. Wang ML, Beeckman-Wagner LA, Wolfe AL, Syamlal G, Petsonk EL. Lung-function impairment among US underground coal miners, 2005 to 2009: Geographic patterns and association with coal workers' pneumoconiosis. J Occup Environ Med. 2013;55:846-50. https://doi.org/10.1097/ JOM.0b013e31828dc985

14. Mo J, Wang L, Au W, Su M. Prevalence of coal worker's pneumoconiosis in China: A systematic analysis of 20012011. Int J Hyg Environ Health. 2014;217:46-51. https://doi. org/10.1016/j.jijheh.2013.03.006

15. Laney AS, Petsonk EL, Hale JM, Wolfe AL, Attfield MD. Potential determinants of coal workers' pneumoconiosis, advanced pneumoconiosis, and progressive massive fibrosis among underground coal miners in the United States, 20052009. Am J Public Health. 2012;102(Supl.2):279-83. https:// doi.org/10.2105/AJPH.2011.300427

16. Rendón ID, Mazuera ME, Grisales H. Neumoconiosis en la minería subterránea, Amagá, 1995. Revista Facultad Nacional de Salud Pública.1997;14:46-67.

17. Orduz C. Neumoconiosis en Colombia, situación en Antioquia. Presentación de 189 casos. Revista Colombiana de Neumología. 2000;13:224-5.

18. Ministerio de la Protección Social. Informe de enfermedad profesional en Colombia. 2007. Fecha de consulta: 7 de febrero de 2017. Disponible en: http://www.istas.net/upload/ Enf\%20profesional\%20Colombia.pdf

19. García-Río F, Calle M, Burgos F, Casan P, del Campo F, Galdiz JB, et al. Espirometría. Arch Bronconeumol. 2013:49:388-401. https://doi.org/10.1016/j.arbres.2013.04.001

20. Barros A, Hirakata V. Alternatives for logistic regression in cross-sectional studies: An empirical comparison of models that directly estimate the prevalence ratio. BMC Med Res Methodol. 2003;3:21. https://doi.org/10.1186/1471-2288-3-21

21. Wade WA, Petsonk EL, Young B, Mogri I. Severe occupational pneumoconiosis among West Virginian coal miners: One hundred thirty-eight cases of progressive massive fibrosis compensated between 2000 and 2009 . Chest. 2011;139:1458-62. https://doi.org/10.1378/chest.10-1326

22. Ministerio de Salud. Indicadores básicos 2012 - Situación de salud en Colombia. Bogotá, D.C.: Minsalud; 2012. p. 61. 
23. Laney AS, Attfield MD. Coal workers' pneumoconiosis and progressive massive fibrosis are increasingly more prevalent among workers in small underground coal mines in the United States. Occup Environ Med. 2010;67:428-31. https://doi.org/10.1136/oem.2009.050757

24. McConnochie K, Green FH, Vallyathan V, Wagner JC, Seal RM, Lyons JP. Interstitial fibrosis in coal workers: Experience in Wales and West Virginia. Ann Occup Hyg. 1988;32:553-60. https://doi.org/10.1093/annhyg/32.inhaled_ particles_VI.553

25. Balmes J, Becklake M, Blanc P, Henneberger P, Kreiss K, Mapp C, et al. American Thoracic Society statement: Occupational contribution to the burden of airway disease. Am J Respir Crit Care Med. 2003;167:787-97. https://doi. org/10.1164/rccm.167.5.787

26. Suarthana E, Laney AS, Storey E, Hale JM, Attfield MD. Coal workers' pneumoconiosis in the United States: Regional differences 40 years after implementation of the 1969 Federal Coal Mine Health and Safety Act. Occup Environ Med. 2011;68:908-13. https://doi.org/10.1136/ oem.2010.063594

27. Centers for Disease Control and Prevention. Coal workers' health surveillance program - data query system. NIOSH: Fecha de consulta: 29 de octubre de 2012.
Disponible en: https://www.cdc.gov/niosh/topics/cwhsp/ecwhsp. $\mathrm{html}$

28. Liu GT, Li XJ, Wei YH, Wei WL, Wang XH, Yuan H, et al. Epidemiological analysis of pneumoconiosis in the Xinjiang Uygur Autonomous Region and cases reported by the Urumqi Railway Bureau. Genet Mol Res. 2015;14:1612-23. https://doi.org/10.4238/2015.March.6.8

29. Xia Y, Liu J, Shi T, Xiang H, Bi Y. Prevalence of pneumoconiosis in Hubei, China from 2008 to 2013. Int $\mathrm{J}$ Environ Res Public Health. 2014;11:8612-21. https://doi. org/10.3390/ijerph110908612

30. Ministerio de Minas y Energía. Censo Departamental Minero, Colombia, julio de 2012. Fecha de consulta: 7 de gebrero de 2017 Disponible en: www.minminas.gov.co/ documents/10180/698204/CensoMinero.pdf/093cec5705e8-416b-8e0c-5e4f7c1d6820

31. Zhang M, Wang D, Zheng YD, DU XY, Chen SY. Analyses on the characteristics and the trends of pneumoconiosis notified between 1997 and 2009, in China. Zhonghua Lao Dong Wei Sheng Zhi Ye Bing ZaZhi. 2013;31:321-34.

32. Perret JL, Plush B, Lachapelle P, Hinks TS, Walter C, Clarke P, et al. Coal mine dust lung disease in the modern era. Respirology. 2017;22:662-70. https://doi.org/10.1111/ resp.13034 